\title{
Pressure effect on the solid-liquid equilibrium forms of even normal alkanes
}

\author{
Koji Shigematsu *, Yoshihiro Terui, Shingo Matsuzaka, Yoshinori Takahashi ${ }^{\dagger}$ \\ Faculty of Education, Iwate University, Morioka 020-8550, Japan
}

\begin{abstract}
This paper describes the changes in equilibrium forms of small even-numbered normal alkane crystals as functions of temperature, pressure and the carbon number of alkane chains. The crystals were grown by increasing pressure of the liquids using a diamond anvil cell (DAC). After single crystals were prepared in the DAC under high pressure, the crystals were maintained for a long period under constant pressure and temperature. During the changes in the shape of the single crystal, a thick plate of $\{001\}$ plane with sharp edges was obtained within a narrow temperature range. The appearance of the thick plate is discussed with reference to the pressure and temperature dependencies of the anisotropy of the surface energy of the crystals.
\end{abstract}

Keywords: Crystal morphology; Solid-liquid equilibrium; Even normal alkanes; Pressure-induced crystal growth; Diamond anvil cell

* Corresponding author. Fax: +81 196216560 .

E-mail address: sigematu@iwate-u.ac.jp (K. Shigematsu).

${ }^{\dagger}$ Present address: Agricultural Environment Department, Iwate Agricultural Research Centre, Narita, Kitakami 024-0003, Japan 


\section{Introduction}

Rod-shaped molecules function widely as structural materials of membranes in living cells. Natural fatty acids, typical rod-shaped molecules, constitute phospholipids which are a main component of the membranes. Most of these have even-numbered carbon chains. In fact, odd-numbered normal alkane molecules cannot overlap themselves, when their chain-directions are reversed. Living cells seem to reject such irreversible carbon chains.

Rod-shaped molecules crystallize into strong anisotropic shapes such as thin plates and needles. We can see that the membranes directly portray the intrinsic shape of rod-shaped molecule assembly. Normal alkane crystals also have thin filmy shapes due to strong anisotropic interactions between the molecules. As even-numbered normal alkanes are simple rod-shaped molecules and a main part of natural fatty acids, evennumbered normal alkane crystals can be considered a simple model of membrane.

Under a uniform driving force for growth, we can observe the growth shape brought about by the anisotropy in growth rate. After a long equilibration with maintenances of constant pressure and temperature, we can observe the equilibrium shape brought about by the anisotropy in interfacial energy. Pressure control in a crystal growth system from liquid can instantly provide a uniform driving force on an entire interface. Accordingly, pressure-induced grown crystals are useful to investigate their anisotropies.

Small even-numbered normal alkanes $(n=8,10,12, n$ : carbon number) are suitable for pressure-induced crystal growth, because they are at liquid phase at room temperature, and are easily solidified by compression. In addition, they have a clear growth unit of one molecule, since they have strong covalent bond in themselves and weak interaction between molecules. Although the equilibrium shapes of even n-alkane crystals reveal their anisotropy in interfacial energy, there are, to date, no reports on the observation of their equilibrium shapes. We thus investigated the equilibrium shape of 
small even n-alkane crystals under uniform pressure.

There are many reports on the crystal structures of n-alkane crystals. Even nalkane crystal series $(6 \leq n \leq 26)$ have a triclinic structure [1-6], in which the lattice constants $a, b$, and the angle $\gamma$ slightly depend on $n$; the lattice constant $c$ and the angles $\alpha$ and $\beta$ strongly depend on $n$, and interactions between molecules along $a$ and $b$ axes are strong and proportional to $n$, and those along $c$ axis are very weak and independent of $n$ (Fig. 1). They are expected to have strong anisotropic equilibrium forms that depend on a strong anisotropy in interfacial energy. From the crystal structures, the equilibrium shapes of even n-alkanes have been theoretically predicted [7].

We observed their equilibrium forms along the liquid-solid equilibrium line by varying both pressure and temperature in a diamond anvil cell (DAC). As the DAC was optimised for optical microscopy [8], we can observe the crystal shapes clearly through the diamonds. The pressure in the DAC becomes uniform quickly due to the coexistence of the crystals and liquids, and due to its small compressed region of a thin disk shape. The temperature of the DAC also becomes uniform, because the upper and lower faces of the compressed region are bounded by diamonds which have the largest thermal conductivity among solids at room temperature. The crystals and liquids in the DAC were thus maintained quiescently for max. 20 days under both constant temperature and pressure.

In our observation, the $\{001\}$ planes of small even n-alkanes were dominant in all forms. Most of these were thin plates bounded by round side faces. In narrow temperature region, they showed thick plate of $\{001\}$ planes with sharp edges. The temperature of the region decreased with increasing $n$. The shape could be explained by the pressure and temperature dependences on the interfacial energy. This crystal shape change will provide the possibility of controllig the morphology of the filmy crystal of normal chain organic compound. The changes may suggest the formation process of the self-organized membrane texture in living cells. 


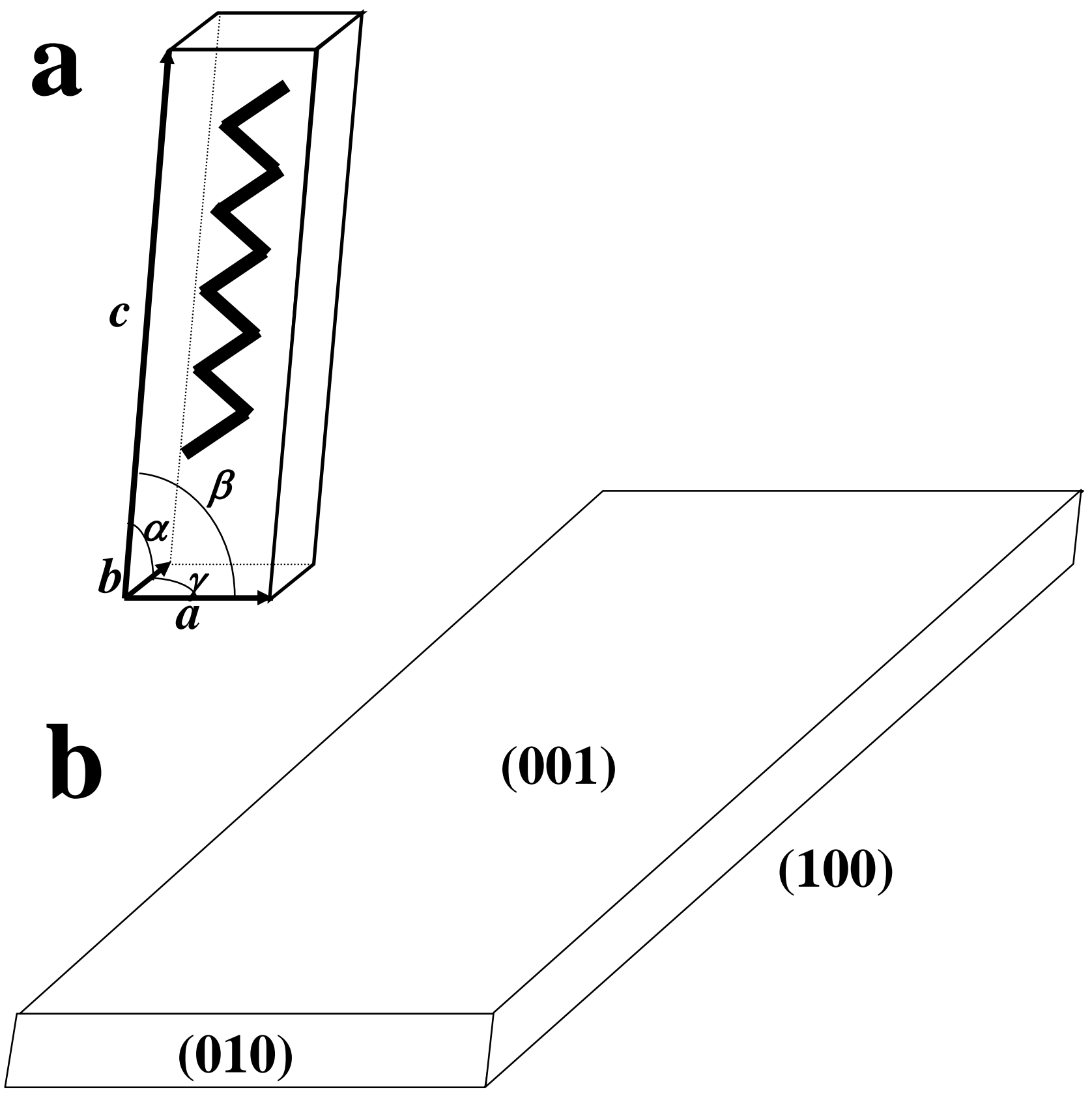

Fig. 1 (a) The triclinic unit cell of the even normal alkane. Here, $a, b$ and $c$ note the crystal axes and the lattice constants of the cell, and $\alpha, \beta$ and $\gamma$ note the angles between the crystal axes. A zigzag line in the cell notes C-C bond chain of even n-alkane. (b) A theoretically predicted equilibrium form of the alkane with indices of crystal surfaces [7]. 


\section{Experimental apparatus and procedure}

Three kinds of small even-numbered normal alkanes (n-octane $\mathrm{C}_{8} \mathrm{H}_{18}$, n-decane $\mathrm{C}_{10} \mathrm{H}_{22}$ and n-dodecane $\mathrm{C}_{12} \mathrm{H}_{26}$ ) of analytical grade (purity $>99 \%$ ) made by Merck Schuchardt OHG were used as they are supplied (without further purification). These even n-alkane liquids were directly drawn from their sample bottles using disposable micro-syringes to prevent unnecessary contamination.

We confined a small amount of the even n-alkane in the hole (diameter: $0.6 \mathrm{~mm}$ ) of a gasket (thickness: $0.5 \mathrm{~mm}$ ), interposed between the upper and lower diamond faces of a diamond anvil cell (DAC). The gasket was made of a Pt-5\%Au alloy which was selected to avoid contamination of the even n-alkane. We directly observed the specimen in the DAC using an inverted optical microscope [9]. After solidification of the entire liquid by compression, a single crystal survived after several pressure adjustments, and was used for our observation.

Pressure was measured by the ruby fluorescence technique with a precision of \pm $0.03 \mathrm{GPa}$ [10]. Thus a ruby ball was enclosed together with the even n-alkanes. Temperature was regulated with a precision of $\pm 0.1 \mathrm{~K}$ by using a water jacket in which temperature-regulating water was flowing.

\section{Results}

Solid-liquid equilibrium pressures as functions of temperature for the even nalkanes are shown in Fig. 2. The pressures of even n-alkanes had linear temperature dependences.

Figure 3 shows the equilibrium forms of the even n-alkanes (n-octane, n-decane and n-dodecane) in the DAC at the temperatures of $10^{\circ} \mathrm{C}, 20^{\circ} \mathrm{C}$ and $30^{\circ} \mathrm{C}$ and at the equilibrium pressures corresponding to these temperatures. The crystal forms shown in Fig. 3 were those of a crystal in a sequence of the pressure and temperature variation. 
The crystal forms changed rapidly just after the pressure and temperature adjustments, slowly changed for 6 hours from the adjustments, and stopped changing after the 6 hours. We confirmed that one of the forms did not change after 480 hours from the adjustment. We thus decided to take the images of the crystals shown in Fig. 3 as the equilibrium forms after 20 hours, since the crystal reaches steady state after 6 hours from the adjustments.

Figure 4 shows the schematic drawings of the equilibrium forms shown in Fig. 3. In Fig. 4, the growth forms are also drawn as parallelograms. The growth forms strongly agreed with the theoretically predicted form [7]. The equilibrium forms of n-octane at $30^{\circ} \mathrm{C}$, n-decane at $20^{\circ} \mathrm{C}$ and n-dodecane at $10^{\circ} \mathrm{C}$ were relatively thick plates of $\{001\}$ plane bounded by $\{100\}$ and $\{010\}$ planes with sharp edges. The other forms were basically thin plates of $\{001\}$ planes with narrow round side faces (complex of $\{100\}$ and $\{010\}$ planes). The thin plates of the n-alkanes also appeared at the temperatures higher than $30^{\circ} \mathrm{C}$ and at temperatures lower than $10^{\circ} \mathrm{C}$. The width of the temperature region in which the thick plate appeared was less than $5 \mathrm{~K}$. 


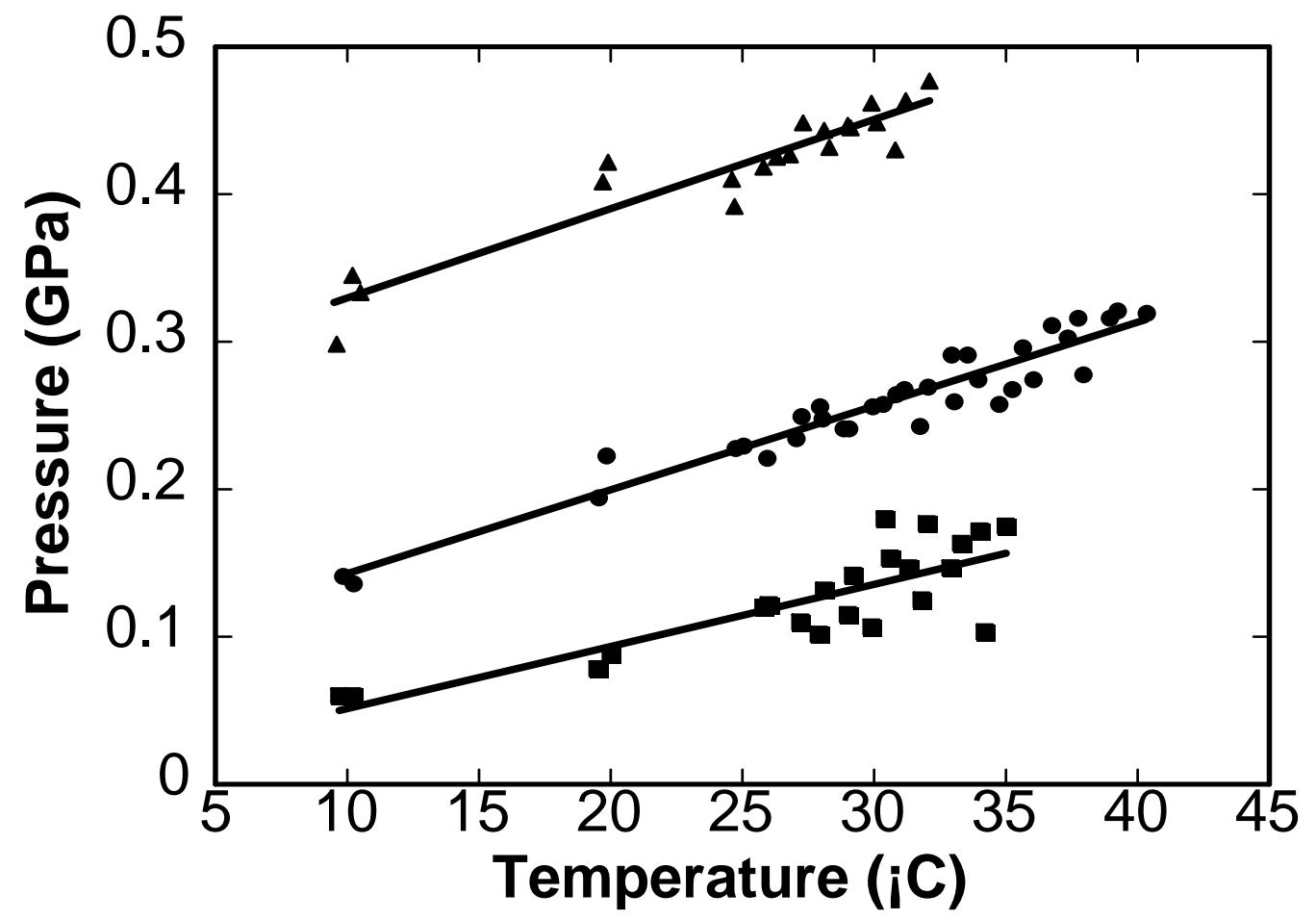

Fig. 2 Temperature dependences of the solid-liquid equilibrium pressures for the even n-alkanes (n-octane: closed triangle, n-decane: closed circle, n-dodecane: closed square). The fitting lines of equilibrium pressure $p(\mathrm{GPa})$ as a function of temperature $T\left({ }^{\circ} \mathrm{C}\right)$ obtained by the method of least squares are as follows: $n$-octane: $p=0.0060 T+0.2706$, n-decane: $p=0.0057 T+0.0861$, n-dodecane: $p=0.0042 T+0.0097$. The correlation coefficients of the lines are 0.941 (n-octane), 0.957 (n-decane) and 0.816 (n-dodecane). 

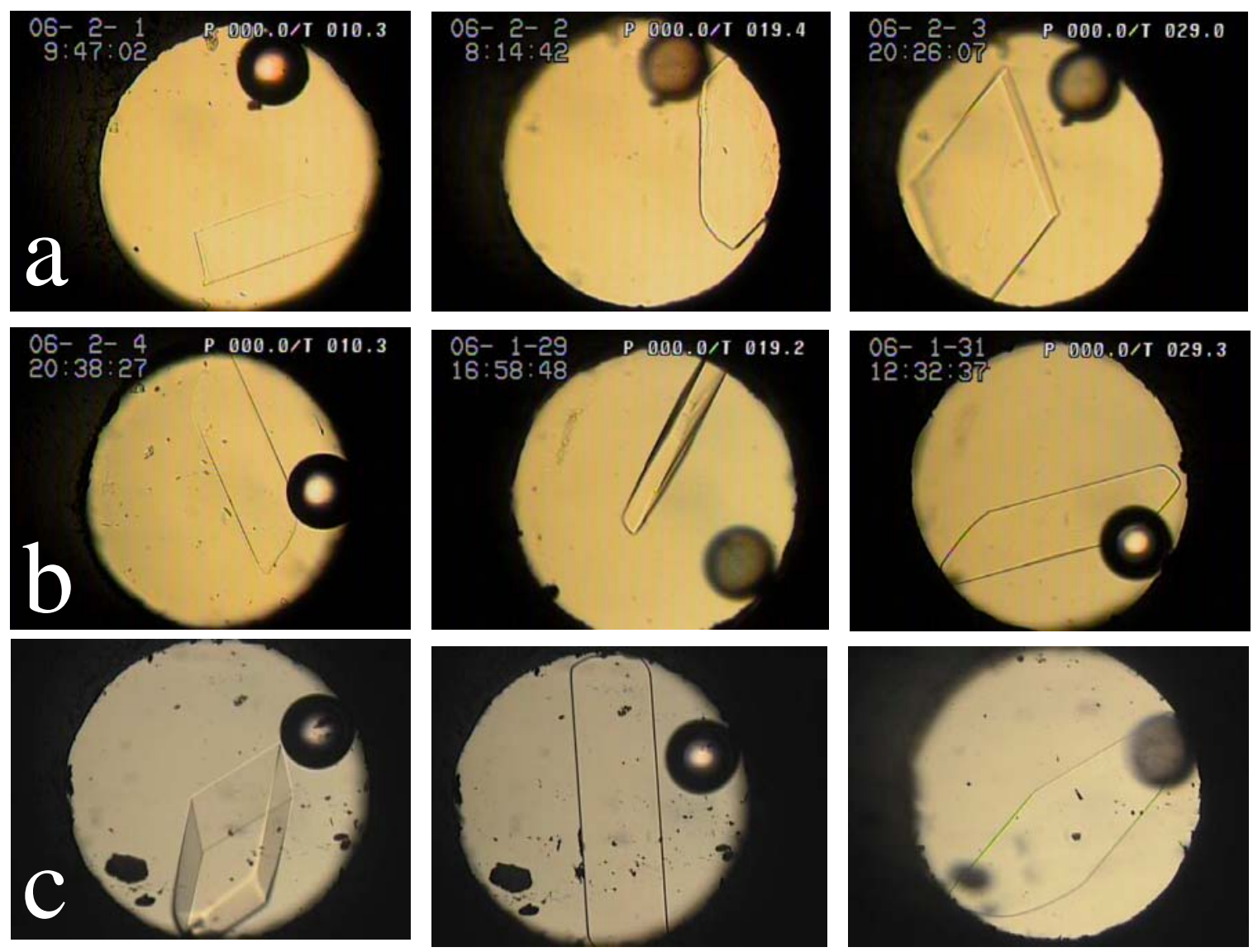

Fig. 3 Images of the equilibrium forms of the alkanes. From the first to the third column, the temperatures are 10,20 and $30^{\circ} \mathrm{C}$ respectively. The rows correspond to the alkanes as follows: (a) n-octane, (b) n-decane and (c) n-dodecane. The sphere in the gasket hole of the DAC is a ruby ball (diameter: $0.134 \mathrm{~mm}$ ) used for pressure measurement. The scale bar indicates $0.1 \mathrm{~mm}$. 

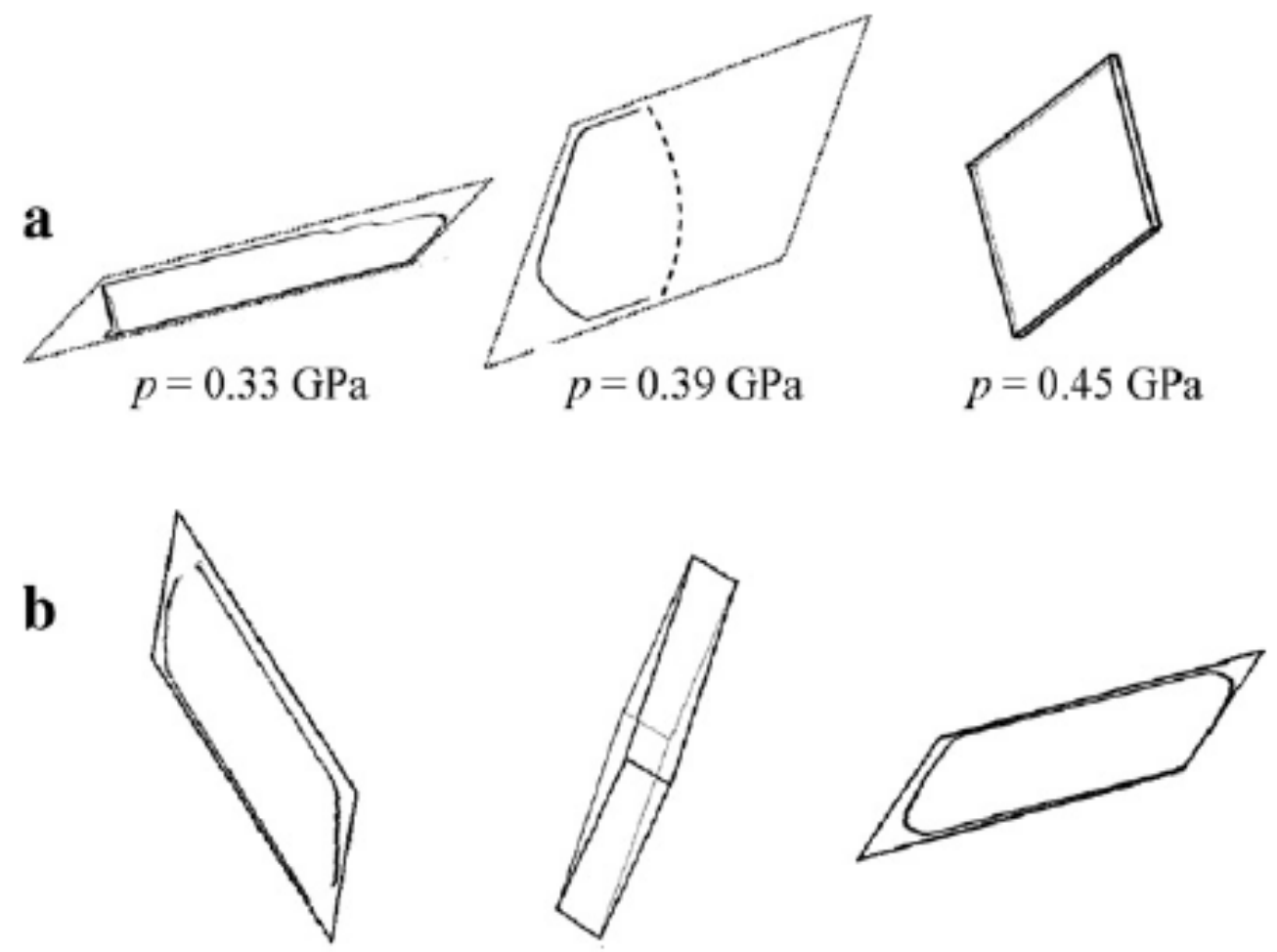

$p=0.14 \mathrm{GPa}$

$p=0.20 \mathrm{GPa}$

$$
p=0.26 \mathrm{GPa}
$$

\section{c}
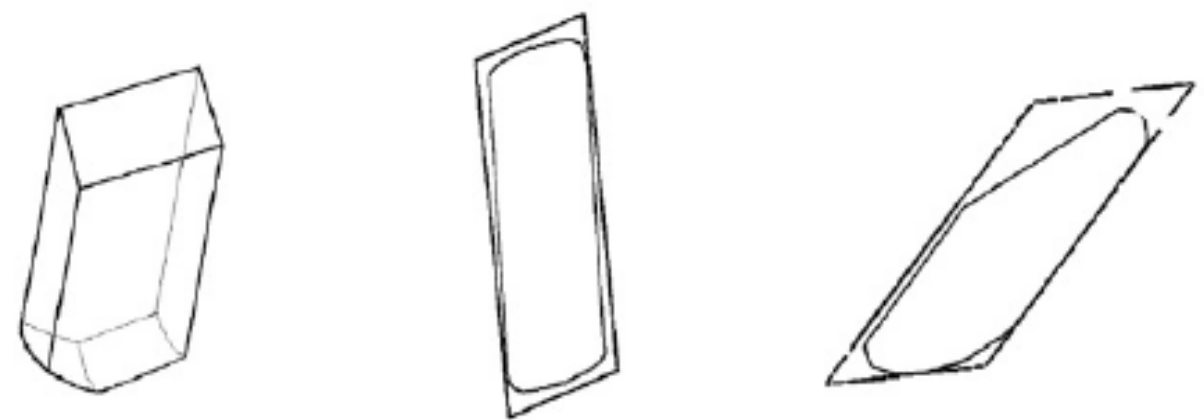

$p=0.05 \mathrm{GPa}$

$p=0.09 \mathrm{GPa}$

Fig. 4 Edges of the equilibrium forms, image-enhanced, of the alkanes shown in Fig. 3. Panel arrangement is the same as that of Fig. 3. The parallelograms which surround the equilibrium forms with broken line note the edges of the growth forms that appeared just after the compression for the next stage. The equilibrium pressure $p$ is indicated in each panel. 


\section{Discussion}

As equilibrium form is only decided by the anisotropy of interfacial energy, the change of equilibrium form should be attributed to that of the anisotropy. The varied parameters of our system were only pressure and temperature. We therefore examined the pressure and temperature dependences of interfacial energy.

Pressure decreases inter-molecule distance. As the decrease in crystal lattice parameters with increasing pressure increases the density of interaction at solid-liquid interface, pressure increases interfacial energy between the molecule on crystal surface and the adjacent liquid molecule.

Pressure, furthermore, increases the anisotropy of interfacial energy, because the decrease in crystal lattice parameters with increasing pressure is not uniform on the entire interface of an even n-alkane crystal. As the compressibility of even n-alkane crystal must have a strong anisotropy identical to that of the interaction between the molecules of the crystal, the decrease in crystal lattice parameters with increasing pressure has a strong anisotropy. The contractions of $a$ and $b$ axes will be larger than that of $c$ axis of even n-alkane, because the $c$ axis is mainly occupied by the strong covalent bond of even n-alkane. We can say that the increase of interfacial energy of $\{001\}$ plane of even n-alkane with increasing pressure is larger than those of $\{100\}$ and $\{010\}$ planes, that is, the increase of the anisotropy of interfacial energy.

On the other hand, temperature increases the randomness of the $\mathrm{C}-\mathrm{C}$ bond chain in the n-alkane. As the random carbon chain decreases the interactions not only between the liquid molecules, but also between the solid and liquid molecules, temperature increase decreases interaction between the molecule on crystal surface and the adjacent liquid molecule.

Temperature, furthermore, decreases the anisotropy of interfacial energy, because the decrease of interaction between the molecule on crystal surface and the adjacent liquid molecule is not uniform on the entire interface of an even n-alkane crystal. The 
\{001\} plane of even n-alkane is the regular arrangement of the terminals of carbon chains. Whereas, the $\{100\}$ and $\{010\}$ planes of even n-alkane are the parallel arrangements of the long sides of carbon chains.

The interaction between the surface molecule on $\{001\}$ plane and the adjacent liquid molecule of the random carbon chain slightly decreases with increasing temperature. This is because the interaction weakly depends on the randomness of the carbon chain due to the point-like interaction between a terminal of carbon chain on $\{001\}$ plane and a small part of the random carbon chain in the liquid molecule.

On the other hand, the interactions between the surface molecules on $\{100\}$ and \{010\} planes and the adjacent liquid molecules of the random carbon chain rapidly decrease with increasing temperature. This is because the interactions strongly depends on the randomness of the carbon chain due to the line-like interaction between the sides of carbon chain on $\{100\}$ and $\{010\}$ planes and a part of the random carbon chain in the liquid molecule of which length rapidly decreases with increasing temperature. Moreover, the difference of the interaction between the sides of carbon chain on $\{100\}$ and $\{010\}$ planes and the random carbon chain increases with increasing temperature due to the difference of the area and the number of exposed carbon atoms in the sides.

Thus, we can say that the decreases of interfacial energies of $\{100\}$ and $\{010\}$ planes of even n-alkane with increasing temperature is larger than that of $\{001\}$ plane, that is, the decrease of the anisotropy of interfacial energy.

\section{Conclusions}

The pressure and temperature effects thus inversely apply to the anisotropy of interfacial energy. The pressure effect prevails over that of temperature in the early stage of the simultaneous increases of pressure and temperature, due to the rapid increase of the density of interaction at solid-liquid interface. The density increases very sensitively by the decrease in crystal lattice parameters with increasing pressure. As 
pressure increases the anisotropy in interfacial energy, the anisotropy between $\{001\}$ plane, and $\{100\}$ and $\{010\}$ planes increases in the early stage. Moreover, the anisotropy between $\{100\}$ and $\{010\}$ planes is small and slightly increases in the early stage. Even n-alkane crystals, thus, show thin plates of $\{001\}$ planes with round side faces.

The temperature effect prevails over that of pressure in the next stage of the simultaneous increases of pressure and temperature due to the slow decrease of the anisotropy in interfacial energy. The anisotropy between $\{001\}$ plane, and $\{100\}$ and $\{010\}$ planes decreases, and the anisotropy between $\{100\}$ and $\{010\}$ planes increases with increasing temperature in the next stage. Even n-alkane crystals, thus, show thick plates of $\{001\}$ planes with sharp edges.

The pressure effect again prevails over that of temperature in the final stage of the simultaneous increases of pressure and temperature, due to the saturation of the temperature effect. The temperature effect is strongly affected by the randomness of the carbon chain. The randomness easily saturates with increasing temperature. Thus, even n-alkane crystals again show thin plates of $\{001\}$ planes with round side faces.

The randomness of the carbon chain, which affects the temperature effect on the anisotropy of interfacial energy, is depended on the number of carbon chain. As even nalkane of a large carbon number has strong interaction between the molecules, the linear configuration of the molecule is maintained even in high temperature under a constant pressure. With a larger number, the linear configuration is maintained at higher temperature under a constant pressure.

The even n-alkane crystals, however, were put under equilibrium conditions where the pressure and the temperature were varied simultaneously along the solidliquid equilibrium lines. As even n-alkane of a large carbon number has many single C$\mathrm{C}$ bonds possible to rotate in the molecule, the linear configuration of the molecule is easily lost even in the low temperature under equilibrium pressure. With the larger 
number, and thus an increase in randomness, the greater the decrease of the anisotropy and thick plates of $\{001\}$ planes with sharp edges appear in the lower temperature under equilibrium pressure.

\section{References}

[1] A. Müller and K. Lonsdale, Acta. Cryst. 1 (1948) 129.

[2] W. M. Mazee, Rec. trav. chim. 67 (1948) 197.

[3] N. Norman and H. Mathisen, Acta. Chem. Scand. 15 (1961) 1747.

[4] H. Mathisen, N. Norman and B. F. Pedersen, Acta. Chem. Scand. 21 (1967) 127.

[5] S.C. Nyburg and H. Lüth, Acta. Cryst. B28 (1972) 2992.

[6] S.C. Nyburg and J.A. Potworowski, Acta. Cryst. B29 (1973) 347.

[7] X.-Y. Liu and P. Bennema, J. Cryst. Growth 135 (1994) 209.

[8] K.Takemura, O. Shimomura and T. Sawada, Rev. Sci. Instrum. 60 (1989) 3783.

[9] K. Shigematsu, T. Sawada, Y. Takahashi and S. Gomi, Jpn. J. Appl. Phys. 38 (1999) L1124.

[10] J. D. Barnett, S. Block and G. J. Piermarini, Rev. Sci. Instrum. 44 (1973) 1. 